\title{
Caracterización de predios productores de arándanos (Vaccinium corymbosum), según nivel tecnológico. El caso de la región del Maule-Chile
}

\author{
Yony M. Ormazábal(1), Carlos A. Mena ${ }^{(1) *}$, Juan C. Cantillana ${ }^{(2)}$ y Germán E. Lobos ${ }^{(3)}$ \\ (1) Centro de Geomática, Facultad de Ciencias Forestales, Universidad de Talca. Avda. Lircay s/n, Talca, Chile. \\ (e-mail: yormazabal@utalca.cl; cmena@utalca.cl) \\ (2) Facultad de Administración y Economía, Universidad Tecnológica Metropolitana. Dr. Hernán Alessandri 722, \\ Providencia, Chile. (e-mail: juan.cantillana@utem.cl) \\ (3) Facultad de Economía y Negocios, Universidad de Talca. Avda. Lircay s/n, Talca, Chile. (e-mail: globos@utalca.cl)
}

* Autor a quien debe ser dirigida la correspondencia

Recibido Abr. 1, 2019; Aceptado Jun. 3, 2019; Versión final Jul. 31, 2019, Publicado Feb. 2020

\section{Resumen}

Se evalúa el Nivel Tecnológico (NT) de los cultivos de arándanos (Vaccinium corymbosum) en la región del Maule-Chile según variables técnicas y características asociadas. Se determinó el NT para cada cuartel de producción utilizando la técnica de Sumatoria Lineal Ponderada y la evaluación de un equipo experto para la asignación de pesos. El procedimiento fue implementado en un Sistema de Información Geográfica, donde además se generó la cartografía temática. Los resultados indican que el $59 \%$ de la superficie de cuarteles de arándanos de la región del Maule-Chile presentan un nivel tecnológico medio (NT2), el resto de la superficie se divide entre los niveles alto y bajo (NT1 y NT3) con un $19 \%$ y $22 \%$, respectivamente. Se concluye que el mayor porcentaje de superficie plantada de arándanos presenta tecnologías medias, apreciándose en las últimas décadas un incremento en la implementación de NT más altos. espacial

\section{Characterization of farm fields of Blueberry (Vaccinium corymbosum) according to technological level. The case of the Maule region, Chile}

\begin{abstract}
The objective of the study was to evaluate the Technological Level (TL) of blueberry crops (Vaccinium corymbosum) in the Maule Region, Chile, according to technical variables and associated characteristics. The TL for each production quarter was determined using the Weighted Linear Combination technique and the evaluation of an expert team for weight assignment. The procedure was implemented in a Geographic Information System, where thematic cartography was also generated. The results indicate that $59 \%$ of the area of blueberry quarters in the Maule Region, Chile, show a medium technological level (TL2), while the rest of the area is divided between high and low levels (TL1 and TL3) with $19 \%$ and $22 \%$, respectively. In conclusion, it is established that the highest percentage of planted area of blueberries presents medium technologies, with an increase in the implementation of higher TL in the last decades.
\end{abstract}

Keywords: blueberries; Vaccinium corymbosum; agroindustry; technological level; geomatics; spatial distribution 


\section{INTRODUCCIÓN}

La producción mundial de arándano en los últimos años ha registrado un importante incremento, mostrando alzas sostenidas por sobre el $5 \%$ en la primera parte de la presente década (Medina y Sánchez, 2014), en tanto que las exportaciones totales a nivel mundial superan las 467.000 toneladas métricas, generando negocios por USD $\$ 1.500$ millones (Peano et al., 2017), situación motivada por el aumento en el consumo de este fruto (Ronceros, et al. 2007). El arándano es una especie del género Vaccinium originaria de Norteamérica, descrita como un arbusto de baja altura, de hoja simple, ovada a lanceolada y caduca (Muñoz, 2015), cuyo fruto es una baya esférica de coloración desde azul claro hasta negro azulado (Vaccinium corymbosum), con un calibre que fluctúa entre los 0,7 a los $1,5 \mathrm{~cm}$ (Stückrath y Petzold, 2007; Külkamp et al., 2014). En Chile, el arándano fue introducido en la década de los ochenta (Bañados, 2006, citado por Pannunzio et al., 2011), siendo su cultivo impulsado como parte de la estrategia de mercado de diversificación de las actividades productivas de la agricultura chilena (Meriño-Gergichevich et al., 2016). Su adaptabilidad a las condiciones geográficas de la zona centro sur de país, lo ha convertido en uno de los productos relevantes de la industria frutícola nacional, registrándose un aumento considerable de la superficie cultivada desde su introducción hasta la actualidad, llegando a ocupar la tercera posición mundial en superficie plantada (Retamales et al., 2014).

El nicho bioclimático donde se desarrolla el arándano corresponde a sectores con temperaturas que fluctúan entre los $7^{\circ} \mathrm{C}$ y los $33^{\circ} \mathrm{C}$, alcanzado su crecimiento óptimo entre los $16^{\circ} \mathrm{C}$ a los $25^{\circ} \mathrm{C}$. Los suelos ácidos son ideales para su crecimiento, con un PH de entre 4,0-5,0; arenosos, franco arenosos o arcillosos; no muy profundos y de baja fertilidad. Estas condiciones favorables se encuentran en la zona centro sur de Chile, especialmente entre los $35^{\circ}$ y $37^{\circ}$ de latitud Sur, que presenta un clima templado mediterráneo con estación seca prolongada, caracterizado por inviernos suaves, primaveras templadas y veranos secos. Hacia 2001, la superficie cultivada con arándanos en Chile alcanzaba las 1.910 ha, la mayoría de las cuales (35,8\%) se encontraba en la región de Los Lagos, al sur del país, en tanto que la región del Maule contaba apenas con el $10,6 \%$ del total nacional. En 2015 las plantaciones de este fruto ocuparon 15.560 ha, el $28 \%$ de las cuales se encontraban en el Maule, región que junto a la del Biobio, concentraron el 56\% del total país (ODEPA, 2015). En este contexto, si bien el proceso ha sido exitoso en cuanto a la creciente superficie ocupada por el cultivo en el país, este ha sido implementado mediante distintos esquemas de producción basados en tecnologías diversas (Bañados, 2009). Este nivel tecnológico está relacionado con la superficie en producción, el año de plantación, las variedades en producción, las técnicas de cultivo (Bryla y Strik, 2006), la asociatividad de productores, entre otros. Particularmente, tiene relación con el material vegetal utilizado y su origen, el uso de mulch (o acolchado) (Cox, 2009), el tipo de sistema de riego (Caron et al., 2017), el diseño de plantación, el uso de camellón, el tipo de control de malezas, el tipo de sistema de producción y el sistema de cosecha (Ganter et al., 2013).

En términos generales, el cultivo del arándano se desarrolla en hileras montadas sobre camellones en el terreno. Los marcos de plantación tienen una alta variabilidad, dependiendo de la especie plantada, el mercado al cual se orienta la producción o las expectativas del productor (Cazanga y Leiva, 2013). Es posible encontrar plantaciones con alta densidad de plantas por hectárea (sobre 6.000) en diseños de $2 \mathrm{~m}$ entre hilera y $0,65 \mathrm{~m}$ entre plantas, hasta sectores con menos de 3.000 plantas por hectárea, con plantaciones de hasta 3,5 m por 1,2 m (entre hilera y plantas, respectivamente) (Holzapfel et al., 2015); situación que incide en la productividad del huerto, dependiendo de la edad de las plantas (Retamales y Hancock 2012). Según las capacidades técnicas y económicas, los camellones son cubiertos por membranas plásticas (mulch) o material orgánico (aserrín u otros), estableciendo formas de riego apropiado para alcanzar los objetivos comerciales del huerto. El material vegetal utilizado en la plantación puede provenir de estacas o cultivos in vitro. Su procedencia generalmente es de un vivero comercial, aunque también es posible que el productor tenga su propio vivero, o que empresas exportadoras distribuyan algunas variedades a sus asociados con el fin de probar su desarrollo en condiciones reales. En este aspecto, las demandas actuales de la industria suponen, entre otros, la necesidad de un recambio varietal que considere la calidad del fruto a producir (González et al., 2013).

La mayoría de las plantas de arándanos entregan frutos al tercer año de plantación, pero es generalmente a partir del cuarto año cuando estos presentan características comerciales. Durante ese tiempo y hasta que la planta sea productiva, es necesario contar con un sistema de control de malezas, siendo el más común el uso de herbicidas (Ganter et al., 2013). A pesar de ello, no son pocos los productores que realizan control manual de malezas, en tanto que otros, utilizan mulch como ayuda para este proceso; situación que puede ser utilizada en forma única o una mezcla de técnicas. De esta forma, la industria del arándano ha ido incorporando herramientas que permiten mejorar el proceso, diferenciándose en el nivel de tecnología aplicada (Matiacevich et al., 2013). Así mismo, la industria ha avanzado en la creación de herramientas que mejoran tanto la eficiencia del proceso, como la condición del trabajador encargado de realizar las labores de remoción de malezas. 
Por su parte, los sistemas de producción reflejan en buena medida el nivel de tecnología aplicado. El sistema de cultivo predominante es el denominado "convencional", que utiliza productos que la empresa agroquímica pone a disposición para mejorar el rendimiento del cultivo y el control de malezas; además del sistema de "producción orgánica" (Becker y Teixeira, 2015), que utiliza mecanismos naturales en el proceso, evitando la incorporación de químicos artificiales en el desarrollo del cultivo (Ganter et al., 2013). En cuanto a la cosecha, los sistemas tradicionales son especialmente sensibles a la productividad individual de los trabajadores involucrados en el proceso, por lo que en el último tiempo se tiende a la incorporación de métodos mecanizados a este proceso (González et al., 2013). De esta forma, los sistemas de producción, independiente de si son convencionales u orgánicos, se pueden caracterizar según el tipo de técnica o método utilizado. Algunas de estas son el cultivo al aire libre, el desarrollado en invernaderos, el uso de túneles, las mallas sombreadoras y también el cultivo en bolsas (Cazanga y Leiva, 2013).

Investigaciones realizadas para obtener un aumento de la productividad de estos cultivos han demostrado las ventajas del uso de sistemas controlados de producción, tales como el invernadero o el uso de mallas sombreadoras (Rodríguez y Morales, 2015), lo que evidencia el aporte de la aplicación tecnológica como elemento de importancia para la industria. Similar situación se produce con los sistemas de regadío, los que han sido utilizados tanto para efectos de aporte del agua necesaria a las plantas como para el control de heladas (Olszewski et al., 2017). Por otra parte, aunque el sistema de cosecha más utilizado en Chile es la cosecha manual, cada vez es mayor la cantidad de huertos que utiliza sistemas de cosecha mecanizados, como vibradoras o cosechadoras automotrices, lo cual significa una ventaja en cuanto a tiempos de cosecha y costo de mano de obra.

En la literatura científica, experiencias como las reportadas por Pannunzio et al. (2011), Battany (2012), Medina y Sánchez (2014) y Holzapfel et al. (2015), demuestran que la incorporación de un mayor nivel de tecnología puede significar un aumento importante en la productividad del cultivo de arándanos. Por tanto, conocer la situación actual en cuanto a los sistemas y tecnologías utilizadas en su cultivo, es un elemento base para gestionar adecuadamente este sector agroindustrial a nivel de región y país. Actualmente, se desconoce el nivel tecnológico implementado en el cultivo de arándanos, lo cual dificulta la adopción e implementación de políticas e iniciativas tendientes a mejorar la calidad de la fruta y el volumen de producción (Rodríguez y Morales, 2015; Meriño-Gergichevich et al., 2016). En este contexto, el presente estudio tiene como objetivo determinar el Nivel Tecnológico (NT) de los cultivos de arándanos de la región del Maule según variables técnicas y características asociadas.

\section{METODOLOGÍA}

El estudio se realizó en la región del Maule, zona que reúne la mayor superficie plantada con arándanos de Chile, alcanzando el $29,6 \%$ del total nacional (ODEPA y CIREN, 2016). Dentro de la región, más del $50 \%$ de las plantaciones de arándano se encuentran en la provincia de Linares. La comuna de Retiro concentra el $15,7 \%$ de las plantaciones a nivel regional con un total de 450,3 ha, seguida por Linares (11\%) y Longaví (11\%), con más de 300 ha cada una. La provincia de Cauquenes contiene un $9,7 \%$ de la superficie plantada a nivel regional, equivalente a 277,9 ha. Por su parte, las provincias de Curicó y Talca presentan menor superficie plantada, destacando las comunas de Romeral, Pencahue y Curepto, con un total de poco más de 100 ha plantadas en las tres comunas.

\section{Características de la zona de estudio}

La región del Maule se encuentra localizada en la macro zona de Chile Central, entre los $34^{\circ} 44^{\prime}$ y $36^{\circ} 32^{\prime}$ de latitud Sur, que corresponde a una sección de territorio nacional caracterizada por un clima mediterráneo con una estación seca prolongada. Las variaciones de temperatura y precipitación al interior de la región reflejan la diferencia entre los sectores con influencia marina y el sector interior, correspondientes a la zona costera y la altura de la cordillera andina en el oriente. La geomorfología regional se caracteriza por la presencia de cinco unidades de relieve, claramente diferenciadas, que se presentan como franjas longitudinales en sentido Este - Oeste, desde la cordillera andina en el sector más oriental, hasta las planicies litorales, al extremo occidental de la región; entre ellas se desarrollan la precordillera o montaña, la depresión intermedia y la cordillera costera (Börgel, 1983). Los huertos productores de arándanos de la región del Maule se concentran principalmente en la unidad depresión intermedia y en los valles intermontanos de la codillera de la costa, en valles perfectamente delimitados por la presencia de ambas cordilleras en sentido longitudinal y valles excavados por los ríos de origen andino en sentido transversal. Esta situación permite el desarrollo óptimo de los cultivos al complementar la deficiencia hídrica que presenta la zona durante el verano con técnicas adecuadas de regadío según el Índice de Thornthwaite (Rodríguez y Morales, 2015). 


\section{Creación de la Base de Datos para el Sistema de Información Geográfica (SIG)}

En el estudio se utilizó un mosaico color SPOTMaps como cubierta base cartográfica. Este mosaico fue generado por imágenes satelitales SPOT-5 ortorrectificadas con resolución espacial de 2,5 m y con nivel de precisión geométrica en localización de 10-15 m (RMSE), lo cual proporciona una escala cartográfica de trabajo de 1:10.000. Se realizó una validación geométrica mediante puntos de control con tecnología GPS (Global Positioning System) para detectar distorsiones producidas por el desplazamiento propio del ajuste de la imagen sobre la topografía del terreno. Además, se realizaron ajustes de los números digitales (DN) de los píxeles para mejora el contraste y visualización de la imagen, mediante el software ERDAS Imagine. Luego, sobre el mosaico se agregó la información vectorial de caminos, hidrografía y curvas de nivel, generada por el Instituto Geográfico Militar (IGM) de Chile, con la finalidad de elaborar cartografía temática base de la zona de estudio.

Con el fin de realizar el estudio con el mayor nivel de detalle posible, se definió la diferenciación de los cultivos de arándanos en base a la unidad mínima de producción, correspondiente a un "cuartel". Este corresponde a un espacio claramente diferenciado tanto por la variedad cultivada, el método de cultivo, las técnicas utilizadas, y generalmente delimitado del resto por las vías de acceso o la conformación del terreno donde se localiza. Se identificaron y digitalizaron cada uno de los cuarteles de producción de arándanos de la región, sobre la imagen del mosaico ortorrectificado. Dicha información fue almacenada como polígonos georreferenciados en una capa temática del software ArcGIS, en formato "shape". Esta capa temática se integró a la cartografía base previamente elaborada con la información vectorial, con el propósito de generar planos a escala local, que luego fueron utilizados por el equipo de terreno para identificar cada cuartel de arándano en la región del Maule. Mediante receptores GPS cartográficos (Trimble GeoXH), se verificó la digitalización realizada sobre el mosaico, registrando además los cambios o diferencias detectadas en la ubicación específica de cada cuartel de arándano.

La recolección de datos específicos de cada cuartel se realizó durante la temporada de cosecha 2015-2016, mediante una ficha diseñada por un panel de expertos que consideró a profesionales y técnicos del área agronómica, directamente relacionados con el cultivo de arándanos, con extensa experiencia en actividades de gestión, producción o investigación. Particularmente, el panel se conformó con profesores de la Facultad de Agronomía de la Universidad de Talca, Prodesales de las provincias de Curicó y Linares, y un Jefe de Huerto de productores, quienes aportaron sus conocimientos en el ámbito productivo del arándano; grupo al que se unieron dos profesionales expertos en Geomática aplicada del Centro de Geomática de la misma universidad. Estos definieron la información necesaria a levantar y la forma en que esta se generaría, de manera de facilitar su traspaso a la base de datos georreferenciada donde luego se realizarían los análisis tendientes a definir los niveles tecnológicos de los cultivos. La información técnica levantada corresponde a variables relacionadas con la variedad de arándanos cultivados y los sistemas y técnicas de producción utilizados. Esta información fue asociada al RUT del productor, de manera de realizar los enlaces de datos necesarios. Asimismo, se distinguió el tipo de propiedad con el fin de establecer diferencias en cuanto al tamaño de la misma. Por último, utilizando el software ArcGIS se integró la cobertura espacial obtenida de la digitalización de los cuarteles sobre el mosaico y su corrección con datos de terreno, con la tabla temática que se obtuvo de las fichas de terreno con la información técnica asociada. El esquema general de esta etapa se presenta en la figura 1.

\section{Cálculo y clasificación del Nivel Tecnológico}

En la determinación del Nivel Tecnológico (NT) de los cultivos de arándanos se consideraron solamente aquellos cuarteles cuya superficie fuese igual o superior a 1 ha. Predios menores a esta superficie, pertenecen a pequeños propietarios, que en su mayoría los manejan como un cultivo complementario, que aporta ingresos extras a las familias, pero no constituyen el principal sustento, por lo que la inversión en tecnología en ellos no es considerable. Los cultivos analizados consideraron 216 predios, en los cuales se identificaron 2.038 cuarteles, que suman 2.866 ha. Las variables técnicas consideradas para el análisis corresponden a: material vegetal, origen del material vegetal, uso de mulch, sistema de riego, diseño de plantación, uso de camellón, control de malezas, sistema de producción, tipo de sistema de producción y sistema de cosecha.

A partir de las variables indicadas, se determinó el valor del NT para cada cuartel, utilizando la técnica de sumatoria lineal ponderada (SLP), mediante lo cual el equipo de expertos asignó a cada variable un peso 0 ponderación (w) expresado en porcentaje según su importancia. Dentro de cada variable se evaluaron alternativas, jerarquizándolas en orden ascendente, donde el mayor valor representó la mayor importancia (ver tabla 1). Para poder integrar variables con diferente número de alternativas, se realizó la normalización de los valores de las alternativas de cada variable mediante la fórmula (1), generando así valores normalizados que oscilan entre 0 y 1 . El método SLP fue implementado según la fórmula (2) en ambiente SIG. Así se completó la evaluación multicriterio (EMC) para estimar un NT para cada cuartel, para luego analizar los resultados dentro del marco territorial correspondiente (Barredo y Bosque, 1998; Mena et al., 2013). 


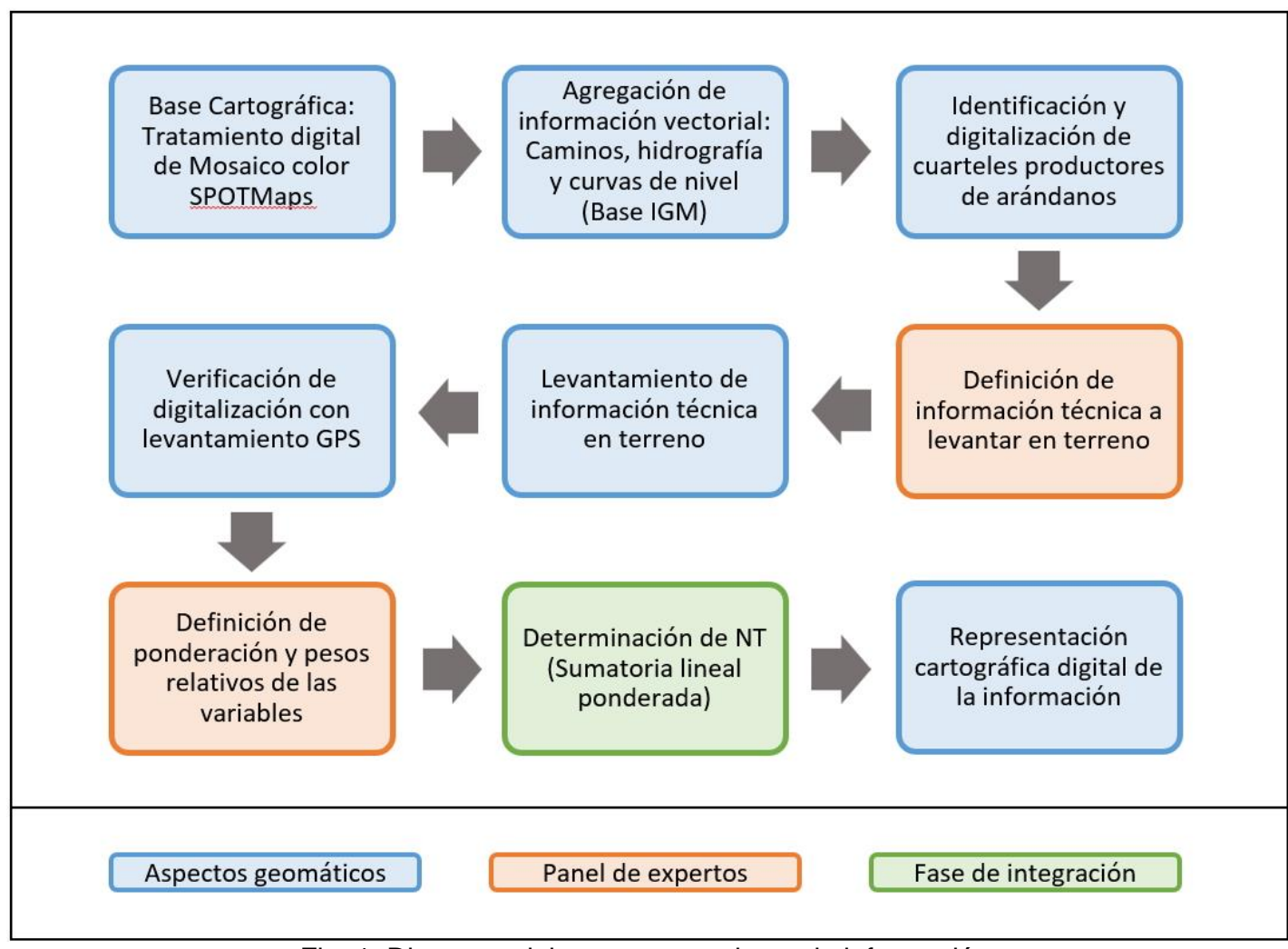

Fig. 1: Diagrama del geoprocesamiento de información.

Tabla 1: Valorización de variables técnico-productivas

\begin{tabular}{|c|c|c|c|}
\hline Variable & $\begin{array}{l}\text { Peso } \\
\text { relativo } \\
\%\end{array}$ & Alternativas & $\begin{array}{l}\text { Valor jerarquizado } \\
\text { (mayor número = } \\
\text { mayor valor) }\end{array}$ \\
\hline Material vegetal & 13,3 & $\begin{array}{l}\text { In vitro } \\
\text { In vitro, estacas } \\
\text { Estacas }\end{array}$ & $\begin{array}{l}3 \\
2 \\
1\end{array}$ \\
\hline Origen del material vegetal & 2,2 & $\begin{array}{l}\text { Vivero comercial } \\
\text { Vivero propio }\end{array}$ & $\begin{array}{l}2 \\
1\end{array}$ \\
\hline Uso de mulch & 20,0 & $\begin{array}{l}\text { Con mulch } \\
\text { Sin mulch }\end{array}$ & $\begin{array}{l}2 \\
1\end{array}$ \\
\hline Sistema de riego & 2,2 & $\begin{array}{l}\text { Goteo } \\
\text { Micro aspersión o micro jet } \\
\text { Surco } \\
\text { Tendido }\end{array}$ & $\begin{array}{l}4 \\
3 \\
2 \\
1\end{array}$ \\
\hline Diseño de plantación & 2,2 & $\begin{array}{l}\text { Bloque completo } \\
\text { Con polinizante }\end{array}$ & $\begin{array}{l}2 \\
1\end{array}$ \\
\hline Uso de camellón & 6,7 & $\begin{array}{l}\text { Con camellón } \\
\text { Sin camellón }\end{array}$ & $\begin{array}{l}2 \\
1\end{array}$ \\
\hline Control de malezas & 15,6 & $\begin{array}{l}\text { Herbicidas } \\
\text { Mixto } \\
\text { Mulch } \\
\text { Manual }\end{array}$ & $\begin{array}{l}4 \\
3 \\
2 \\
1\end{array}$ \\
\hline Sistema de producción & 15,6 & $\begin{array}{l}\text { Orgánico } \\
\text { Convencional }\end{array}$ & $\begin{array}{l}2 \\
1\end{array}$ \\
\hline Técnica de producción & 11,1 & $\begin{array}{l}\text { Malla sombreadora } \\
\text { Invernadero } \\
\text { Bolsas } \\
\text { Aire libre }\end{array}$ & $\begin{array}{l}4 \\
3 \\
2 \\
1\end{array}$ \\
\hline Sistema de cosecha & 6,7 & $\begin{array}{l}\text { Mecanizada } \\
\text { Mecanizada y manual } \\
\text { Manual }\end{array}$ & $\begin{array}{l}3 \\
2 \\
1\end{array}$ \\
\hline
\end{tabular}




$$
v_{i j}=\left(a_{i}-\min a_{j}\right) /\left(\max a_{j}-\min a_{j}\right)
$$

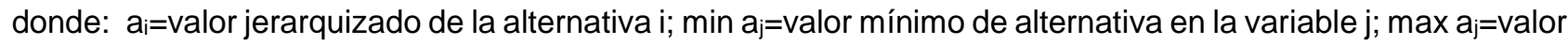
máximo de alternativa en la variable j.

$$
N T_{k}=\sum_{i}^{n}\left(w_{j}^{*} v_{i j}\right)
$$

donde: $\mathrm{NT}_{\mathrm{k}}=$ Nivel Tecnológico para el cuartel $\mathrm{k} ; \mathrm{w}_{\mathrm{j}}=$ peso de la variable $\mathrm{j} ; \mathrm{v}_{\mathrm{ij}}=\mathrm{valor}$ normalizado de la alternativa i en la variable j; $n=$ número de variables involucradas en la investigación.

Los valores algebraicos del NT obtenidos para cada cuartel de arándanos se agruparon en 3 clases de igual amplitud: NT1 (nivel tecnológico alto), NT2, (nivel medio) y NT3 (nivel bajo). Aquí los niveles más altos corresponden a los cuarteles de arándanos que presentan un mayor grado de modernización reflejado en la implementación de técnicas y sistemas productivos más eficientes, según la valoración del equipo de expertos.

\section{Análisis contextual de NT según características asociadas}

Se analizaron aspectos productivos relevantes, asociados a los cuarteles de arándano, con el propósito de contextualizar el comportamiento y distribución de los NT en la zona de estudio. Para esto se agruparon y analizaron los resultados, considerando los siguientes aspectos: superficie de producción, variedades en producción, año de plantación y asociatividad:

Superficie de producción: El conjunto de productores de arándanos se clasificó en tres categorías de acuerdo a la superficie plantada de arándanos de cada propietario, definiendo como T1 a grandes productores (superficie $>20$ ha), T2 a medianos productores (5-20 ha) y T3 a pequeños productores (superficie $<5$ ha).

Variedades en producción: Se definieron tres categorías según la superficie plantada por variedad, de lo cual se estableció $\mathrm{P} 1$ como variedades con los mayores porcentajes de superficie plantada a nivel regional $(52,8 \%$ en conjunto), P2 como variedades con nivel medio de porcentajes de superficie regional plantada (30,2\% en conjunto), y P3 con nivel bajo de porcentajes de superficie regional plantada (17\% en conjunto). De este modo, se clasifican en P1 las variedades de: Brigitta, Duke, Legacy y O'Neal. En P2 se encuentran variedades como: Aurora, Bluecrop, Bluegold, Brightwell, Elliott, Jewel, Liberty, Misty, Ochlockonee, Ozarkblue, Powderblue, Star y Tifblue. En P3 se encuentran variedades como: Alapaha, Berkeley, Biloxi, Bluecrisp, Bluegem, Bluejay, Blueray, Bonita, Camellia, Chandler, Choice, Corona, Costa, Coville, Denisse, Draper, Earliblue, Rebeld, Sierra y Vermont.

Año de plantación: Se clasificaron tres períodos según el año de plantación de cada cuartel, declarado por los productores y administradores. Se tomó como referencia tres décadas significativas para la producción de arándano, definiendo A1 a los cultivos de arándanos plantados hasta el año 1990, A2 a los cultivos de arándanos plantados entre 1991 y 2000, ambos inclusive, y A3 a los cultivos de arándanos plantados desde el año 2001 en adelante.

Asociatividad: Se identificaron dos grandes grupos de productores, G1 productores asociados y $\mathrm{G} 2$ productores no asociados. Los productores asociados participan activamente dentro de una organización donde pueden intercambiar conocimientos junto con nuevas ideas de implementación tecnológica y de desarrollo productivo. Los productores no asociados, no tienen ninguna participación en sociedades u organizaciones productivas.

Por último, se realizó un análisis de las características antes descritas en función de su localización en el territorio regional, considerando para ello en primer lugar la delimitación de carácter político administrativo correspondiente a la división provincial y comunal al interior de la región, y posteriormente un análisis espacial en función de la ubicación de los huertos respecto de elementos de importancia en el territorio, tal como las principales localidades urbanas y las vías de comunicación. Las primeras se consideraron en función de su rol de proveedoras de insumos, soporte para actividades administrativas y financieras, así como principales proveedoras de mano de obra para las plantaciones; mientras que las segundas se consideraron atendiendo a su importancia en el traslado de la cosecha hacia los puntos de distribución interna o exportación.

\section{RESULTADOS Y DISCUSIÓN}

Los resultados obtenidos del análisis de las variables estudiadas, considerando el total de los cuarteles de arándano en la región del Maule, se presentan en la tabla 2. Es posible apreciar que el tipo de material vegetal 
utilizado en las plantaciones de arándano, en su mayoría, es de estaca con el $54,5 \%$, mientras que un $45,5 \%$ corresponden a plantas in vitro, lo cual es bajo considerando que esta es la forma más efectiva de incorporar ejemplares selectos dentro de las áreas cultivadas. El origen del material vegetal, en su gran mayoría, proviene de vivero comercial con el $87,7 \%$, en tanto que vivero propio presenta el $12,3 \%$, lo que sería consistente con la necesidad de recambio varietal que hoy presentan estos cultivos (González et al., 2013). Cox (2009) estudió la relación entre el uso de mulch y la productividad en los cultivos, destacando su efecto positivo. La revisión de los NT en los huertos de la región del Maule indica un alto nivel de adopción de esta técnica, siendo utilizado por el $60 \%$ en los cuarteles de arándano; de estos, el tipo de mulch predominante es polietileno con un $95 \%$ de la superficie, mientras que el $5 \%$ restante es con aserrín. Para el caso de camellón, el $95 \%$ de los cuarteles lo utiliza, el $5 \%$ restante está sin camellón. Asimismo, los cuarteles que controlan maleza con herbicida representan al $6 \%$, mientras que el $10 \%$ lo hace manual y el $5 \%$ con mulch; la mayor proporción, $79 \%$ de los cuarteles, utiliza un sistema mixto de control de maleza. El diseño de plantación por bloque corresponde a un $86 \%$, mientras que un $14 \%$ incorpora polinizantes.

En cuanto al régimen hídrico, el principal origen del agua utilizada es de pozo con un $56,2 \%$, seguido por río, estero o canal con el $42,3 \%$, el $1,5 \%$ restante es por embalse o vertiente. El sistema de riego predominante es por goteo con un $98,61 \%$ y en un menor porcentaje de micro aspersión o microjet con $0,27 \%$, tipo tendido con $0,85 \%$ y surco con $0,27 \%$. Esto se condice con lo planteado por Caron et al. (2017), respecto de la importancia que el sistema de regadío tiene para la productividad de estos cultivares, haciendo hincapié en la necesidad de la optimización de este recurso. La situación a nivel regional presenta un alto índice de aplicación de tecnología, dejando apenas sobre el 1\% de los cuarteles de producción utilizando sistemas de regadío ineficientes.

Tabla 2: Porcentaje de componentes y técnicas productivas, cultivos de arándanos en la región del Maule

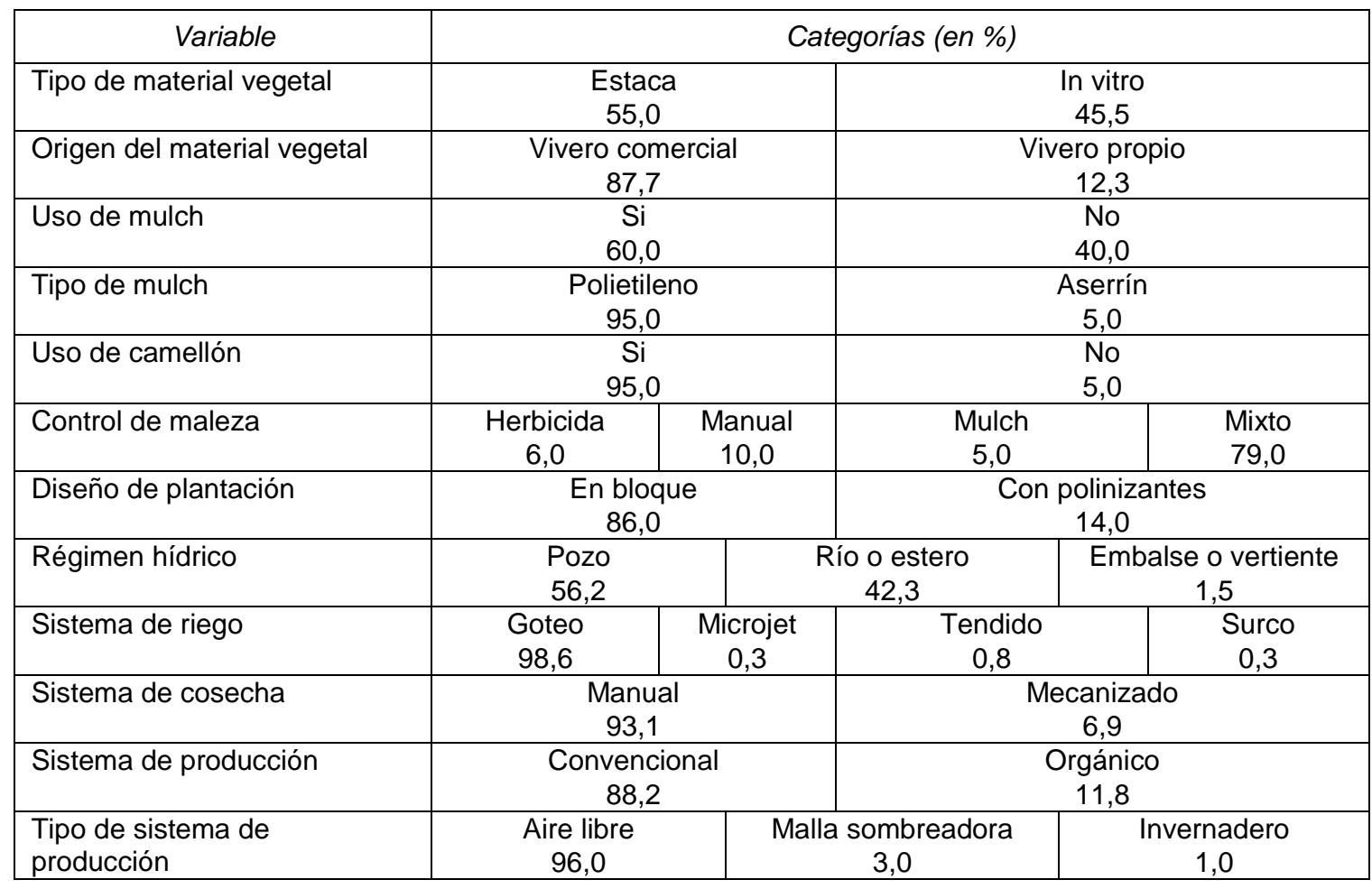

El sistema de cosecha utilizado mayoritariamente es de tipo manual, implementado en un $93,7 \%$ de la superficie plantada, mientras que solo en un $6,3 \%$ usa sistema mecanizado. En cuanto al sistema de producción, el $88,17 \%$ del total de los arándanos presente en la región se encuentra bajo un sistema de tipo convencional, solo un $11,83 \%$ corresponde a manejo orgánico. Respecto del sistema de producción y a pesar de las diversas técnicas de plantación disponibles actualmente (Cazanga y Leiva, 2013), el tipo predominante es al aire libre con un $96 \%$, mientras la malla sombreadora corresponde al $3 \%$ y en invernadero solo al $1 \%$, lo que denota un bajo nivel tecnológico en este aspecto.

\section{Distribución espacial del Nivel Tecnológico}

A partir de la valorización de las variables técnico-productivas según los criterios del grupo de expertos, se obtuvo el Nivel Tecnológico (NT) para cada uno de los cuarteles de arándanos de la región del Maule. Los valores clasificados dentro de las 3 categorías definidas, indican que en la región del Maule existe una superficie de 546 ha (19\%) dentro de la categoría NT1, 1700 ha (59\%) en la categoría NT2 y 619 ha (22\%) 
en la categoría NT3. En términos de distribución espacial según límites político-administrativos (ver figura 2), la provincia de Linares concentra la mayor cantidad de hectáreas plantadas con arándano en la región con 1.704 ha, equivalente al 59\% de la superficie, mientras que la provincia de Cauquenes solo tiene 45 ha, Curicó 497 ha y la de Talca 387 ha. En todas las provincias de la región del Maule predomina el Nivel Tecnológico NT2.

Asimismo, se observa en la figura 1 una tendencia a la concentración de los cuarteles con mayor NT en los alrededores de las ciudades principales, predominando los de menor nivel en aquellas concentraciones poblacionales de menor jerarquía dentro del contexto urbano regional. Similar situación se aprecia respecto de la cercanía a las vías de comunicación, encontrándose una concentración de predios de mayor NT en las proximidades de las carreteras principales, mientras que se denota un predominio de los niveles de NT inferiores en los cuarteles más apartados de estas vías.

\section{NT según características asociadas}

De acuerdo al tamaño de producción: Los medianos productores concentran la mayor superficie plantada de arándanos en la región con un 59\%, mientras que los grandes productores ocupan el $19 \%$ y los pequeños productores el $22 \%$. Se registra la presencia de una superficie cercana al $20 \%$ que está siendo manejada con NT1 considerando todas las categorías de producción. La figura 3 presenta la relación entre NT y T donde se evidencia una disminución en la cantidad de superficie manejada con NT1 a medida que disminuye T, pasando de $10,15 \%$ en T1 a 7,77\% en T2 y solo $0,97 \%$ en T3. Para NT2, la tendencia es similar, con $33,36 \%$ en T1, 23,53\% en T2 y 2,76\% en T3; mientras que para NT3 la mayor concentración de productores corresponde a T2 (10,55\%), luego T1 con 8,14\% y finalmente T3 con solo 2,77\% del total. Es posible observar que para los tamaños de producción de T1 y T2, la mayor cantidad de superficie de cultivo está siendo manejada con NT2; mientras que en T3 la mayor superficie registrada está siendo manejada con NT3 $(2,77 \%)$, seguido de NT2 (2,76\%) y por último NT1 (0,97\%), lo cual indica una tendencia a disminuir el nivel tecnológico a medida que disminuye el tamaño de superficie de producción.

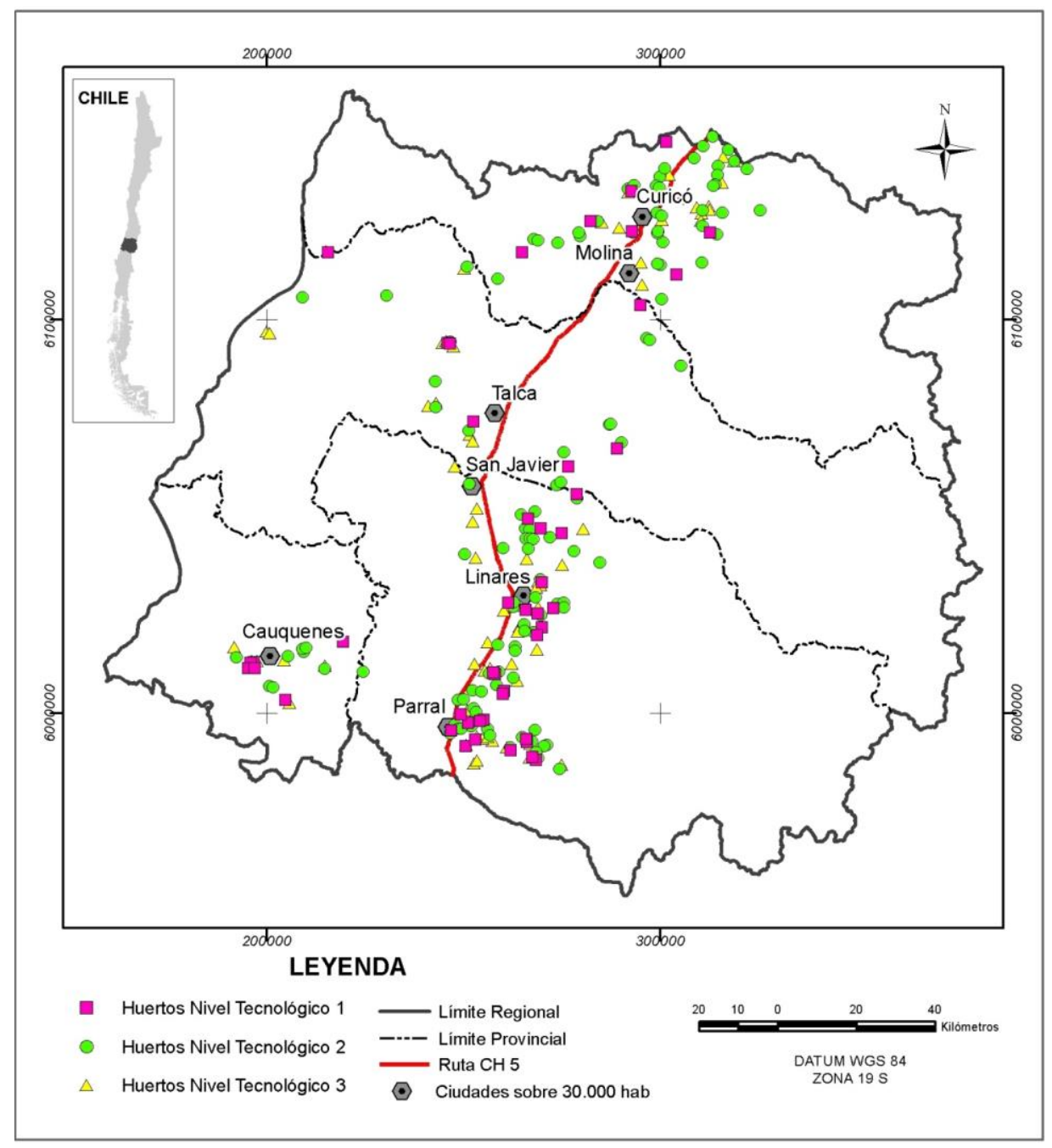

Fig. 2: Distribución de cultivos de arándanos según nivel tecnológico. 


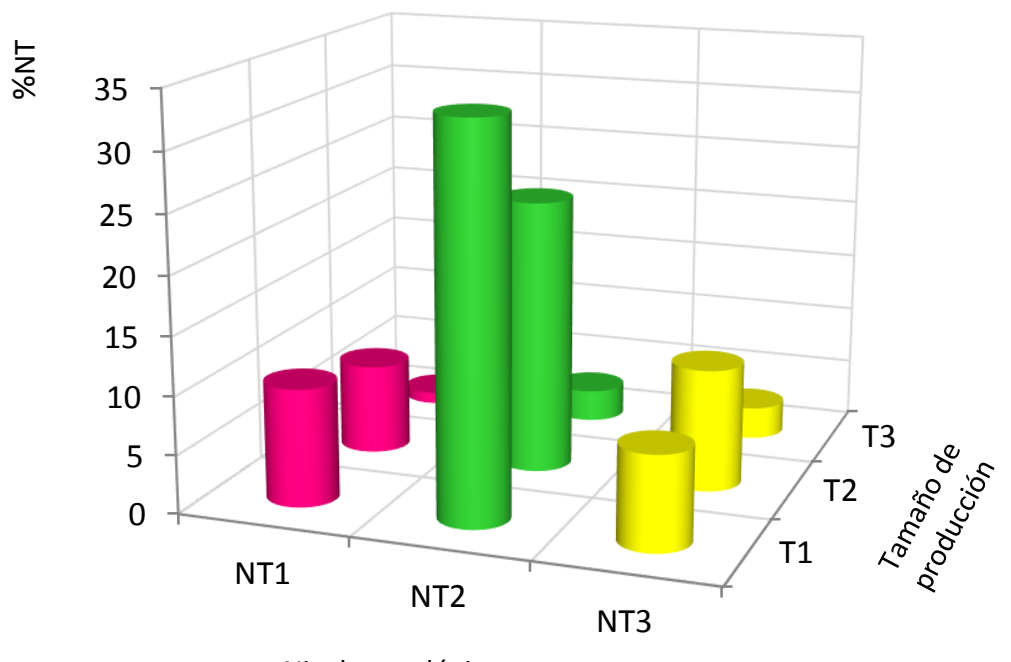

Nivel tecnológico

Fig. 3: Cultivo de arándanos según nivel tecnológico y tamaño de producción.

De acuerdo a las variedades en producción: Se observa que para NT1 la mayor proporción de superficie se presenta en cultivos P1 (11,22\%) seguido por P2 $(5,13 \%)$ y P3 $(2,57 \%)$ (ver figura 4$)$. Para NT2, se presenta una tendencia parecida con valores de $28,34 \%, 20,91 \%$ y $10,37 \%$, para P1, P2 y P3, respectivamente. En tanto, NT3 se concentra en P1 (12,34\%), seguido de P3 $(5,45 \%)$ y P2 (3,68\%).

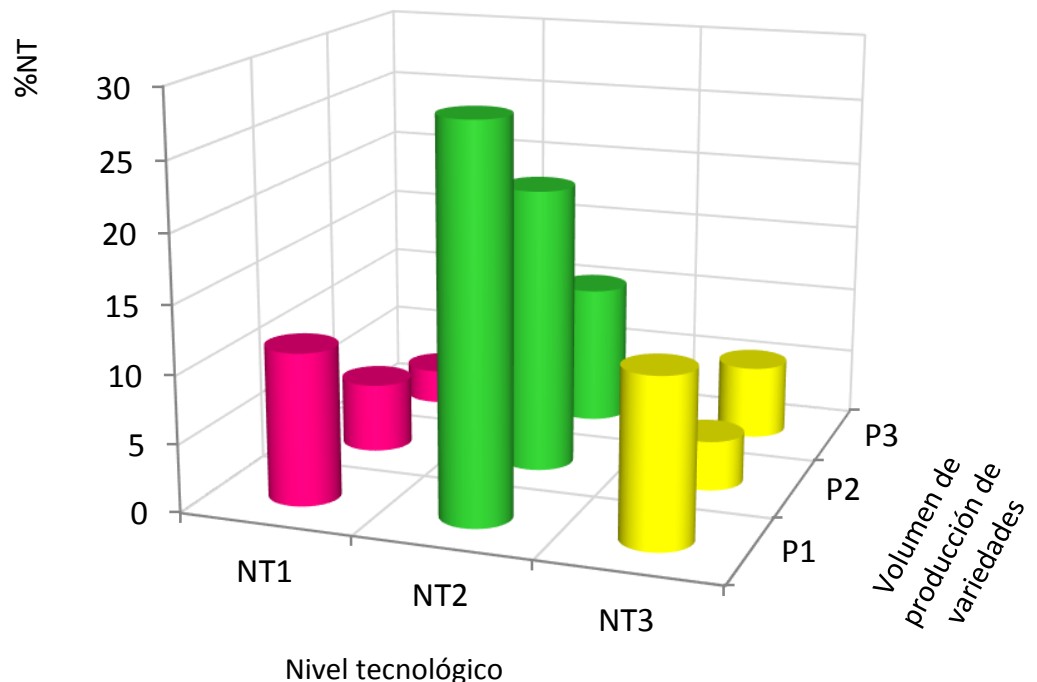

Fig. 4: Cultivos de arándanos según nivel tecnológico y volumen de producción por variedades.

De acuerdo al año de plantación: Los productores que iniciaron su cultivo antes de 1990 (A1) representan solo el $1 \%$ de la superficie plantada, todos ellos manejados en la actualidad con NT2 (ver figura 5). Las plantaciones A2 (establecidas entre 1991 y 2000), representan el $4 \%$ de la superficie donde el 2,20\% de los cultivos se manejan con NT2. El mayor porcentaje de plantación se registra después del 2001 (A3) con un $95,02 \%$ de la superficie total, en este periodo nuevamente el NT2 tiene la mayor implementación con un $56,43 \%$ de la superficie. Esta relación entre NT y periodos de plantación también permite identificar la tendencia a la incorporación de tecnología a través del tiempo, ya que entre las plantaciones realizadas desde 2001 (A3) se observa un alto porcentaje de NT1 y NT2 (18,24\% y 56,43\%, respectivamente), en tanto que los huertos con NT3 alcanzan un $20,36 \%$. Asimismo, cabe hacer notar que de las plantaciones realizadas antes de 1991 ninguna registra manejo con NT1 ni con NT3.

Asociatividad: El 59\% de los productores de arándanos en la región pertenecen a una organización productiva. En los cultivos que presentan NT1, en las tres categorías de tamaño (T1, T2 y T3), tienden a pertenecen a una organización. En los cultivos que implementan NT2 y NT3, los grandes productores tienden a no estar asociados, mientras que los medianos productores si pertenecen a alguna asociación. En cultivos con NT3, la tendencia de estar asociados es similar entre los grandes y pequeños productores, mientras que en los 
medianos productores la tendencia es igual, pero más marcada. En figura 6 se observa que, según el tamaño de producción, los grandes productores (T1) que están asociados, presentan mayor concentración de NT2 $(5,43 \%)$, seguido por NT1 (2,66\%) y NT3 $(1,54 \%)$. Por el contrario, los productores más pequeños (T3) que están asociados, presentan mayor presencia de NT3 y NT2, con $5,22 \%$ y $4,77 \%$, respectivamente, y solo un $1,51 \%$ con NT1. En tanto, los productores T3 no asociados, presentan cifras similares para NT3, NT2 y NT1 (4,35\%, $4,28 \%$ y $1,09 \%$, respectivamente), lo que estaría indicando que para este grupo (T3) no hay diferencia en la implementación de tecnología según estén o no asociados.

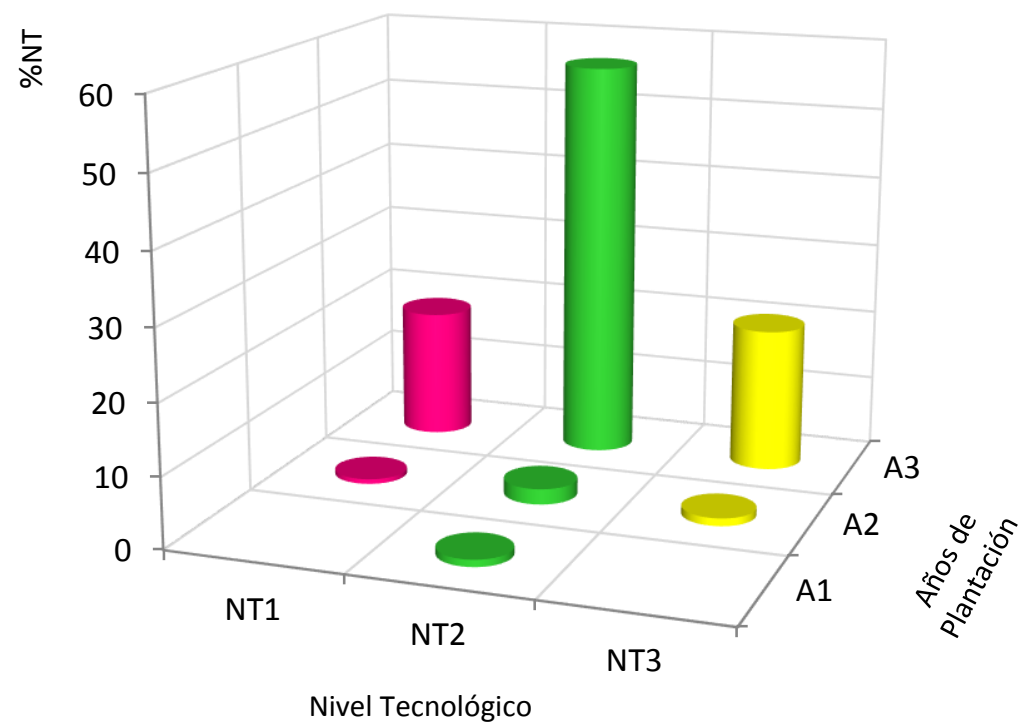

Fig. 5: Cultivos de arándanos según nivel tecnológico y año de plantación.

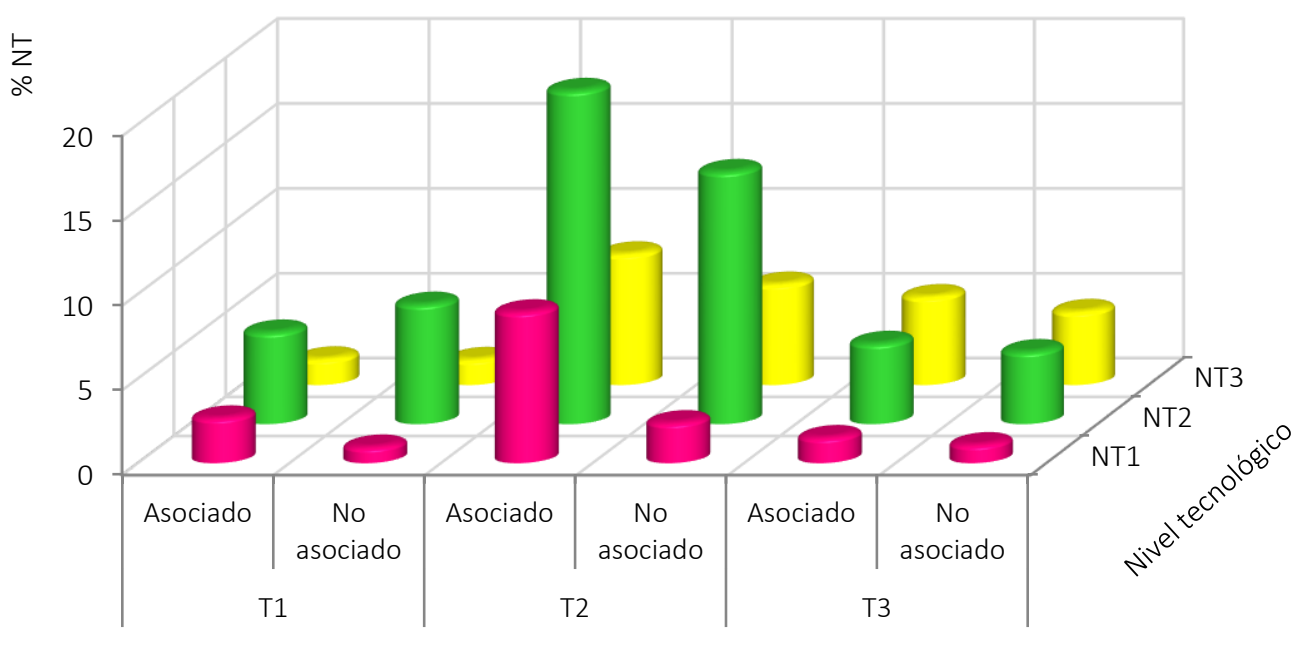

Asociatividad por tamaño de producción

Fig. 6: Asociatividad según tamaño de producción y nivel tecnológico.

Como se observa, en términos generales la implementación de NT en los huertos productores de arándanos, presenta marcadas tendencias en relación a plantaciones más recientes, así como en los tamaños de los huertos productores y variedades de mayor producción. El predominio de NT2 refleja un sector en desarrollo, que ya cuenta con una base productiva importante, pero que aún presenta oportunidades de crecimiento y mejora. Esta situación es concordante con lo expuesto en el informe de Diagnóstico y Prospectiva de la región del Maule, elaborado en el proceso de actualización de la Estrategia Regional de Desarrollo (ERD) del Maule, donde se identifica al sector frutícola y particularmente al cultivo de berries como uno de los ocho sectores económico-productivos relevantes para la región. Dentro de los aspectos prioritarios a trabajar, se menciona el desarrollo de programas de I+D para fruticultura, así como profundizar en sistemas de inteligencia de mercados y potenciar la red de extensionismo para Pymes (GORE Maule, 2007). 
Por su parte, Retamales y Sepúlveda (2011) reconocen que, si bien la producción frutícola se posiciona como un sector exitoso de la economía durante las últimas décadas, se han evidenciado algunas complicaciones relacionadas con aspectos como el efecto negativo en la rentabilidad provocada por menores tasas de cambio, el aumento en los costos de energía, y la mayor escasez y costo de la mano de obra; situaciones que pondrían en riesgo el futuro de una proporción importante de esta industria. El hecho de que un $45 \%$ de la fruta que produce el país es exportada como fruta fresca, obliga a mejorar la eficiencia de los procesos de producción, así como la investigación sobre el particular; aspectos en los que la ERD concuerda plenamente al mencionar que las diferencias que actualmente se encuentran en los rendimientos asociados al manejo de los huertos, pueden estar relacionadas con la generación y transferencia de conocimiento y tecnología.

Retamales et al. (2014) indican como puntos críticos para la industria de arándanos la necesidad de incorporar prácticas de producción sostenibles y más productivas, mejorar la calidad y consistencia de la fruta producida, además de aumentar la inversión en investigación e innovación, entre otros. Detectar carencias en los sistemas productivos y en los aspectos sociales relacionados, como nivel educacional y habilidades técnicas, resulta de gran importancia, sobre todo en lugares donde existen condiciones biofísicas adecuadas que no se aprovechan apropiadamente o que todavía no han sido incorporados al cultivo. Estos aspectos están directamente relacionados con el nivel tecnológico asociado a los cultivos de arándanos, por lo que las metodologías aplicadas y los resultados obtenidos en el presente estudio, pueden apoyar la toma de decisiones en dichas materias. En este sentido, datos georreferenciados respecto del nivel tecnológico de los cultivos pueden orientar las inversiones a realizar en el sector, optimizando la disponibilidad de recursos y maximizando el efecto socioeconómico de la industria de arándanos en el territorio.

\section{CONCLUSIONES}

De acuerdo a las variables técnico-productivas registradas para cada cuartel de arándanos de la región del Maule, se puede indicar que, a modo general, los cultivos de arándanos de la región del Maule corresponden a unidades productivas donde el tipo de material vegetal utilizado mayoritariamente es de estaca, que son plantas provenientes de vivero comercial, que utilizan camellón y mulch de polietileno, con diseño de plantación por bloque, donde el pozo es la fuente principal de regadío seguido por río, estero o canal, cuyo sistema de riego predominante es por goteo, y donde el tipo de cosecha es manual y son cuarteles con producción convencional al aire libre. La evaluación del Nivel Tecnológico en base a las características asociadas indica que el mayor porcentaje de superficie plantada de arándanos presenta tecnologías medias, apreciándose en las últimas décadas un incremento en la implementación de Niveles Tecnológicos más altos, cuyo objetivo sería el de mejorar la productividad de los cultivos. De esta forma, el $59 \%$ de la superficie de los cuarteles de arándanos de la región tiene un nivel medio NT2, mientras que el resto de la superficie se divide entre las categorías NT1 y NT3, con un $19 \%$ y $22 \%$, respectivamente.

\section{REFERENCIAS}

Bañados, M. P., Expanding Blueberry Production into Non-Traditional Production Areas: Northern Chile and Argentina, Mexico and Spain, Acta Horticulturae, 810, 439-445 (2009).

Barredo, J. y J. Bosque, Multicriteria Evaluation Methods for Ordinal Data in a GIS environment, Geographical Systems, 5, 313-327 (1998).

Battany, M., Vineyard Frost Protection with Upward-blowing Wind Machines, Agricultural and Forest Meteorology, 157, 3948, (2012).

Becker, P. y M. Teixeira, Biotransformation in Temperate Climate Fruit: A Focus on Berries, American Journal of Food Science and Technology, 3(4), 12-17 (2015).

Börgel, R., Geomorfología. Colección Geografía de Chile, 1ª Ed., 182. Instituto Geográfico Militar, Santiago, Chile (1983).

Bryla, D. y B. Strik, Variation in Plant and Soil Water Relations Among Irrigated Blueberry Cultivars Planted at Two Distinct in-row Spacing, Acta Horticulturae, 715, 295-300 (2006).

Caron, J., V. Pelletier y otros seis autores, Guidelines of Irrigation and Drainage Management Strategies to Enhance Cranberry Production and Optimize Water use in North America, Canadian Journal of Soil Science, 97(1), 7-8 (2017).

Cazanga, R. y C. Leiva, Antecedentes Técnicos y Económicos para la Producción de Berries y Pistacho en la Región del Maule, $1^{a}$ Ed., 109. Publicación CIREN N¹74, Santiago, Chile (2013).

Cox, J., Comparision of Plastic Weedmat and Woodchip Mulch on Low Chill Blueberry Soil in New South Wales, Australia, Acta Horticulturae, 812, 475-482, (2009).

Ganter, A., R. Montalba, R. Rebolledo y L. Vieli, Plastic Mulch Effects on Ground Beetle Communities (Coleóptera: Carabidae) in an organic blueberry field, Idesia, 31(4), 61-66 (2013).

GORE Maule, Informe de Diagnóstico y Prospectiva. Proceso de Actualización ERD Maule 2008-2020, 1a Ed., 260. Talca, Chile (2007). 
González, A., J. Subercaseaux y M. Ellena, Arándanos: Optimización de la Productividad de la Mano de Obra y Tecnologías para el Incremento de Calidad y Condición en el Sur de Chile, $1^{a}$ Ed., 110. Boletín INIA N²77, Santiago, Chile (2013).

Holzapfel, E., J. Jara y A. Coronata, Number of Drip Laterals and Irrigation Frequency on Yield and Exportable Fruit Size of Highbush Blueberry Grown in a Sandy Soil, Agricultural Water Management, 148, 207-212 (2015).

Külkamp, A. L., R. Rodrigues y otros cinco autores, Produção e Qualidade de Frutos de Mirtileiros sob Diferentes Intensidades de Poda, Ciência Rural, 44(12), 2157-2163 (2014).

Matiacevich, S., D. Celis y otros tres autores, Quality Parameters of Six Cultivars of Blueberry Using Computer Vision, doi:10.1155/2013/419535, International Journal of Food Science, vol. 2013, Article ID 419535, 8 (2013).

Medina, M. y M. Sánchez, Producción y Exportación de Arándanos para Estados Unidos, Tesis Magíster en Administración de Empresas, $1^{\text {a }}$ Ed., 133. Universidad Peruana de Ciencias Aplicadas, Lima, Perú (2014).

Mena, C., Y. Moreno y otros cuatro autores, Caracterización del Nivel Tecnológico de Cuarteles Vitícolas en la Comuna de Sagrada Familia, Chile, Interciencia, 38(3), 194-201 (2013).

Meriño-Gergichevich, C., E. Pacheco y M. Reyes-Díaz, Effect of Foliar Boron Spraying on the Fruit Features of Brigitta and Legacy Highbush Blueberry (Vaccinium corymbosum) Cultivars, Ciencia e Investigación Agraria, 43(3), 452-463 (2016).

Muñoz, R., As Condições de Trabalho na Nova Produção de Exportação de Frutas: o Caso de Mirtilo na Província Argentina de Entre Rios, 2002-2010, Trabajo y sociedad, 25, 371-386 (2015).

ODEPA Y CIREN, Catastro Frutícola. Principales Resultados Región del Maule, $1^{a}$ Ed., 50. Publicado por la Oficina de Estudios y Políticas Agrarias del Ministerio de Agricultura y el Centro de Información de Recursos Naturales, Santiago, Chile (2016).

ODEPA, Panorama de la Agricultura Chilena, $1^{\text {a }}$ Ed., 138. Publicado por la Oficina de Estudios y Políticas Agrarias del Ministerio de Agricultura, Santiago, Chile (2015).

Olszewski, F., P. Jeranyama, C. Kennedy y C. DeMoranville, Automated Cycled Sprinkler Irrigation for Spring Frost Protection of Cranberries, Agricultural Water Management, 189, 19-26 (2017).

Pannunzio, A., F. Vilella, P. Texeira y A. Premuzik, Impacto de los Sistemas de Riego por Goteo en Arándanos, Revista Basileira de Engenharia Agrícola e Ambiental, 15, 3-8 (2011).

Peano, C., V. Girgenti, C. Baudino y N. Giuggioli, Blueberry Supply in Italy: Management, Innovation and Sustainability, doi:10.3390/su9020261, Sustainability, 9, 261 (2017).

Retamales, J., M. Palma y otros cuatro autores, Blueberry Production in Chile: Current Status and Future Developments, Rev. Bras. Frutic. Jaboticabal SP, 36(1), 58-67 (2014).

Retamales, J. y J. Sepúlveda, Fruit Production in Chile: Bright Past, Uncertain Future, Rev. Bras. Frutic., Jaboticabal - SP, Volume Especial, 173-178 (2011).

Retamales, J. y J. Hancock, Blueberries, $1^{\text {a }}$ Ed., 323. CABI, Wallingford, UK (2012).

Rodríguez, M. y D. Morales, Shading Nets Effect on the Production and Quality of Blueberry Fruit (Vaccinium corymbosum L.) cv. Brigitta, Scientia Agropecuaria, 6, 41-50 (2015).

Ronceros, B., R. Quevedo y I. Leiva, Efecto de un Pretratamiento Químico en el Deshidratado del Arándano por Métodos Combinados, Información Tecnológica, 18(6), 57-64 (2007).

Stückrath, R. y G. Petzold, Formulación de una Pasta Gelificada a partir del Descarte de Arándanos, Información Tecnológica, 18(2), 53-60 (2007). 